\title{
ADAPTIVE THERMO-COMPENSATION IN MAGNETO-OPTICAL RECORDING
}

\author{
Hideki Saga, Hirofumi Sukeda, Masahiko Takahashi and *Takehiko Yorozu \\ Central Research Laboratory, Hitachi, Ltd., \\ 1-280. Higashi-Koigakubo, Kokubunji-shi, Tokyo 185, Japan \\ *Recording Media Research Laboratory, Hitachi Maxell, Ltd., \\ 6-20-1 Kinunodai, Yawara-mura, Tsukuba-gun, Ibaraki 300-24, Japan
}

\begin{abstract}
In the mark-edge recording system with small marks, it has been difficult to simultaneously optimize an equalizer and recording conditions. So we studied how to optimize these conditions independently in such a system. Then we found out the necessity of compensation for the thermal interference according to not only the length of the preceding space but also to the length of the preceding mark. Therefore we developed Adaptive Themo-Compensation (ATC), which adaptively compensates for the thermal interference depending on the preceding mark length. By ATC, all edgeshifts are suppressed with optimal equalizer within $8.4 \%$ p p of a $0.27-\mu \mathrm{m}$ detection window and, therefore, highly accurate mark-edge controllability is possible. As a result, wide recording power margin is secured.

KEYWORDS: MAGNETO-OPTICAL RECORDING, MARK-EDGE RECORDING, INTER-SYMBOL INTERFERENCE (ISI), THERMAL INTERFERENCE, THERMAL ACCUMULATION, ADAPTIVE THERMOCOMPENSATION (ATC)
\end{abstract}

\section{INTRODUCTION}

The mark-edge recording method is effective for high-linear-density recording [1]. When marks much smaller than the optical spot are formed, the shape of the marks deviates from the ideal depending on the data pattern. So it is difficult to carry out mark-edge recording with small marks. To solve this problem, many methods have been devised [2][3][4]. But, in such a system with small marks, optical inter-symbol interference (ISI) is not negligible. The reproduced signal suffers from both deviation of mark shapes and ISI. Thus, it has been difficult to simultaneously optimize the equalizer that cancels ISI and the recording conditions (such as waveform and power) that determine the shape of the marks. So we studied how to optimize the recording conditions in such a system without an equalizer.

\section{EXPERIMENTS AND RESULTS}

The energy irradiated onto the recording layer to form a mark overflows into the position of the succeeding mark, and the shape of the succeeding mark deviates according to the preceding space length. In a magneto-optical recording system, the smaller the space becomes, the larger the succeeding mark grows. This is called "thermal interference". We found out by thermal simulation that the mark shape is never influenced by the formation of the succeeding mark. Hence, the edge-shift, $\Delta \mathrm{E}_{1}$ in Fig. 1 (shift of the trailing edge of the preceding mark) comes only from ISI. And

$\Delta \mathrm{E}_{2}$ in Fig. 1 (shift of the leading edge of the succeeding mark) comes from both ISI and thermal interference.

When a long mark is formed, the longer the mark becomes, the wider it becomes. This is called "thermal accumulation". Similarly, by the thermal simulation, we found out that the leading edge never moves if the succeeding part is formed. Consequently, the edge-shift, $\Delta \mathrm{E}_{1}$ in Fig. 2 (shift of the leading edge) comes only from ISI. And $\Delta \mathrm{E}_{2}$ in Fig. 2 (shift of the trailing edge) comes from both ISI and thermal accumulation.

Hence, without an equalizer, the recording waveform can be constructed so that the equation $\Delta E_{1}=-\Delta E_{2}$ always holds. Taking the above consideration into account, we built the recording waveform. Table 1 summarizes the parameters of the system in which we evaluated the recording waveform. Then, we evaluated the recording power margin of this waveform for various recording patterns. The results are shown in Fig. 3. Although a wide recording power margin is obtained for a well-randomized pattern such as an incremental pattern, the margin for other particular patterns cannot be secured. For convenience, the worst of 


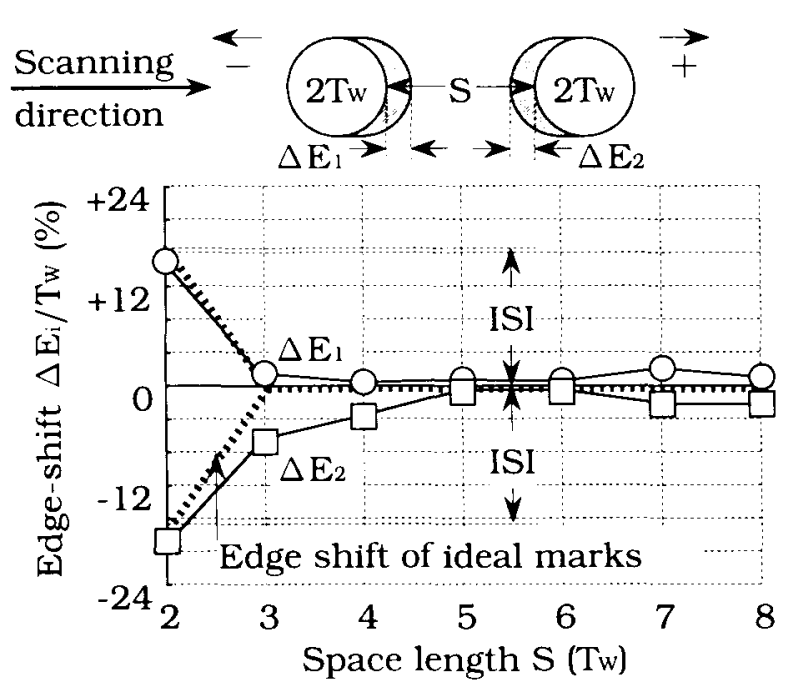

Fig. 1: Dependence of edge-shift on the space length between two marks. For ideal marks, $\Delta E_{1}=-\Delta E_{2}$.

these patterns will be called the "worst pattern" hereafter.

Careful analysis of the edge-shift behavior revealed that the degradation results from the edge-shift deviation shown by the solid symbols in Fig. 4. That is, when two marks are formed closely, the energy to form a mark flows into the position of the succeeding mark. If the preceding mark length is fixed, (namely, if the thermal interference is constant), the thermal interference will be well compensated only by the thermal shut-down period immediately after the preceding mark. But the mark size will deviate according to thepreceding mark length, even though the distance between two marks is fixed. This isbecause the thermal conduction to the position of the succeeding mark varies according to the amount of energy forming the preceding mark. This is explained that the thermal conduction from the preceding part of a mark, in addition to that from the trailing edge of the previous mark, influence the succeeding mark.

As a result, it is necessary to compensate for the thermal interference according to not only the
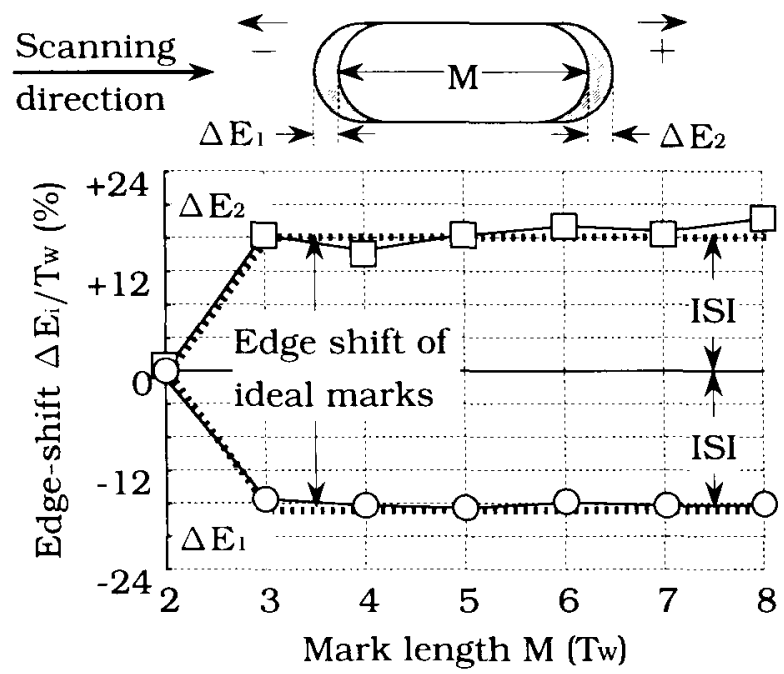

Fig. 2: Dependence of edge-shift on the selfmark length. For ideal marks, $\Delta E_{1}=-\Delta E_{2}$.

\begin{tabular}{|c|c|}
\hline Linear density & $0.40 \mu \mathrm{m} / \mathrm{bit}$ \\
\hline Channel bit length & $0.27 \mu \mathrm{m}$ \\
\hline Modulation & $(1,7) \mathrm{RLL}$ \\
\hline Wavelength $(\lambda)$ & $685 \mathrm{~nm}$ \\
\hline Numerical aperture (NA) & 0.55 \\
\hline Spot diameter $(\equiv \lambda / \mathrm{NA})$ & $1.2 \mu \mathrm{m}$ \\
\hline
\end{tabular}

Table 1: Summary of system parameters. The shortest mark is about $43 \%$ of an optical spot.

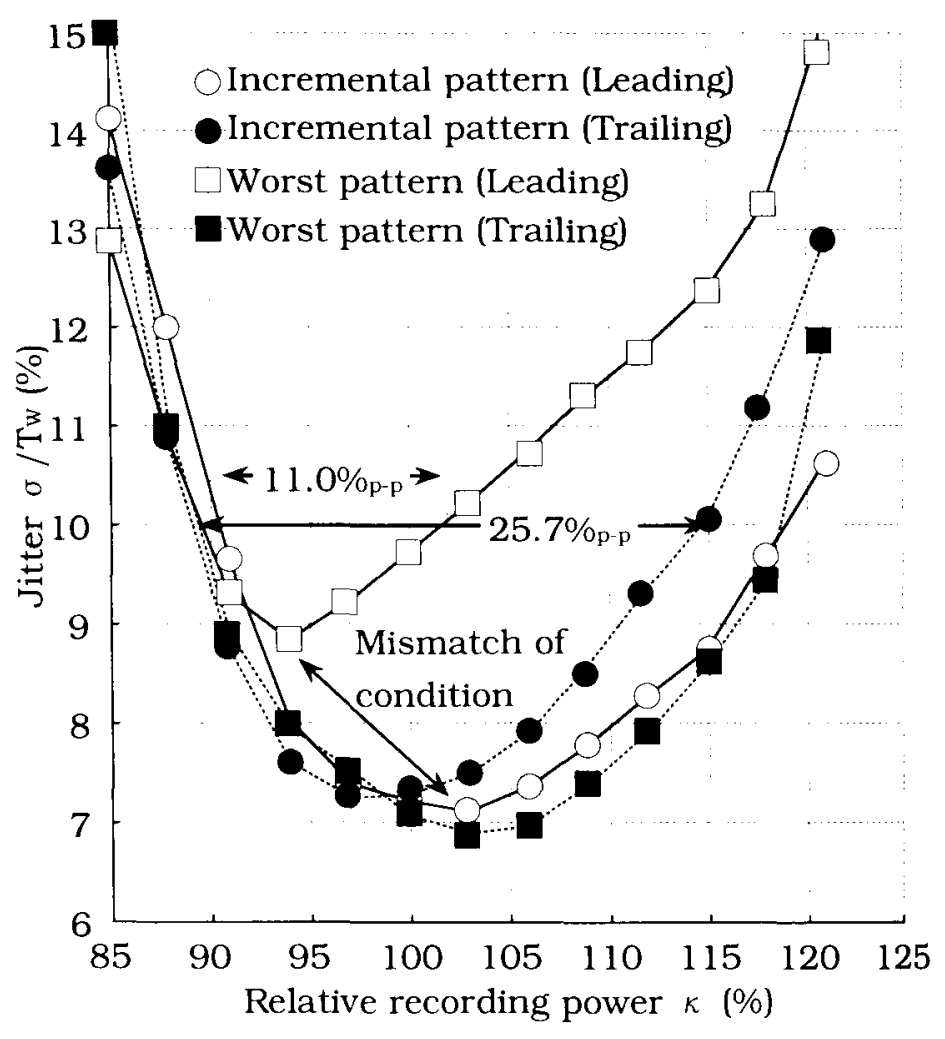

Fig. 3: Recording power margin of non-ATC waveform. The margin for the worst pattern cannot be secured. 


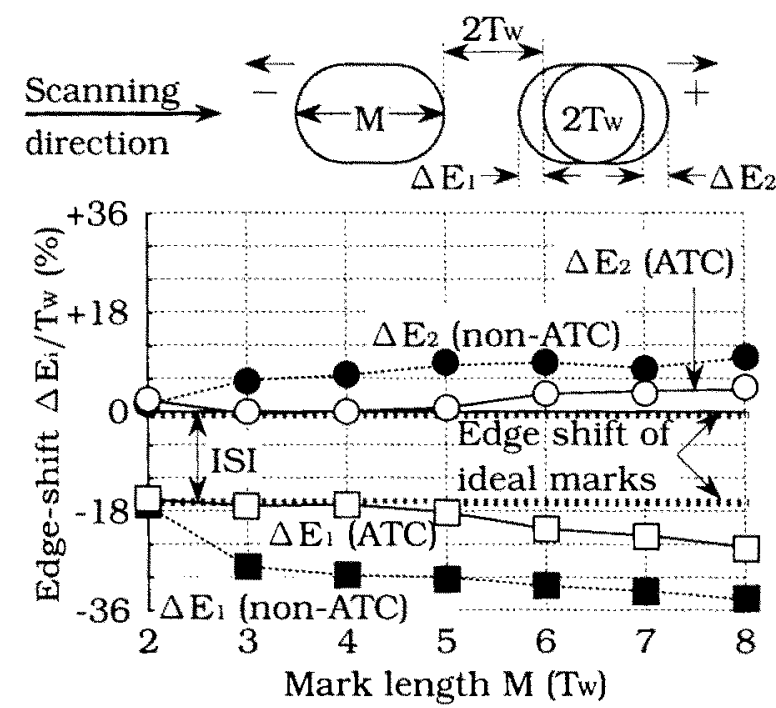

Fig. 4: Dependence of edge-shift on the preceding mark length. For ideal marks, $\Delta E_{1}$ should be constant and $\Delta E_{2}$ shoud be zero.

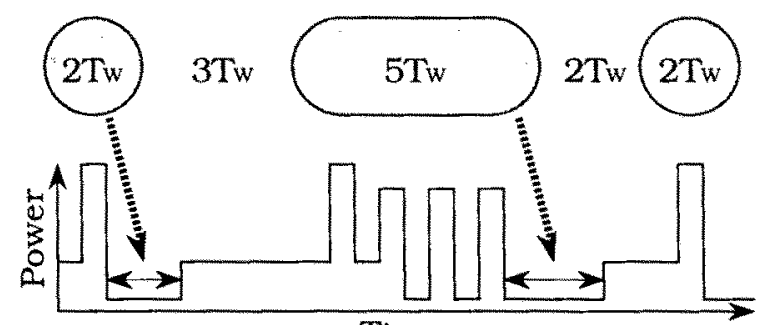

Time

Fig. 5: Recording waveform of Adaptive Thermo-Compensation (ATC). Shut-down period is $1.5 \mathrm{Tw}$ after the $2 \mathrm{Tw}_{\mathrm{w}}$ mark. Otherwise, shut-down period is $2.0 \mathrm{Tw}$.

length of the preceding space but also to the length of the preceding mark. Therefore we developed Adaptive Themo-Compensation (ATC), which adaptively compensates for the thermal interference depending on the preceding mark length. As shown in Fig. 5 , the thermal shutdown period will be changed for the compensation. The shut-down period is $1.5 \mathrm{Tw}$ after the $2 \mathrm{Tw}$ mark. ( $\mathrm{T} w$ is the width of detection window.) Otherwise, the shut-down period is 2.OTw. The edge-shift with ATC is shown in Fig. 4 by open symbols. The edge-shifts are well controlled. Then edge-shift dependence of the leading edge on recording pattern (ie. preceding mark-length and preceding space-

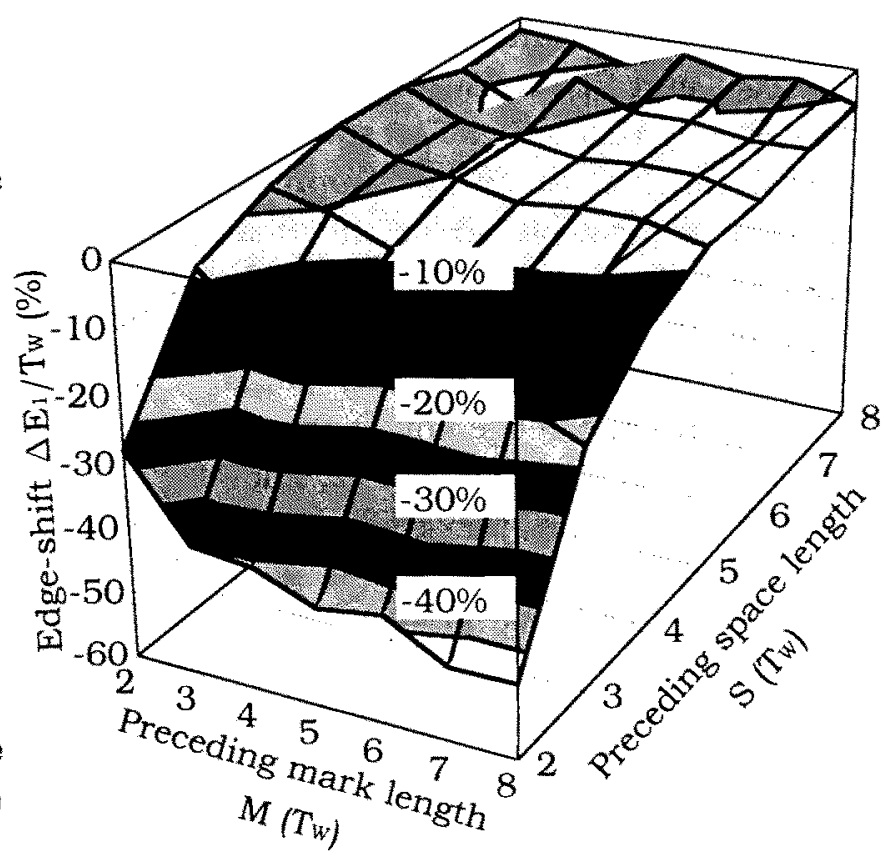

Fig. 6: Leading edge-shift of succeeding mark recorded by non-ATC waveform (without equalizer). Ideal $\Delta E_{1}$ after $2 T w$ space should be constant and not depend on preceding mark length. Otherwise $\Delta E_{1}$ of ideal marks should be zero.

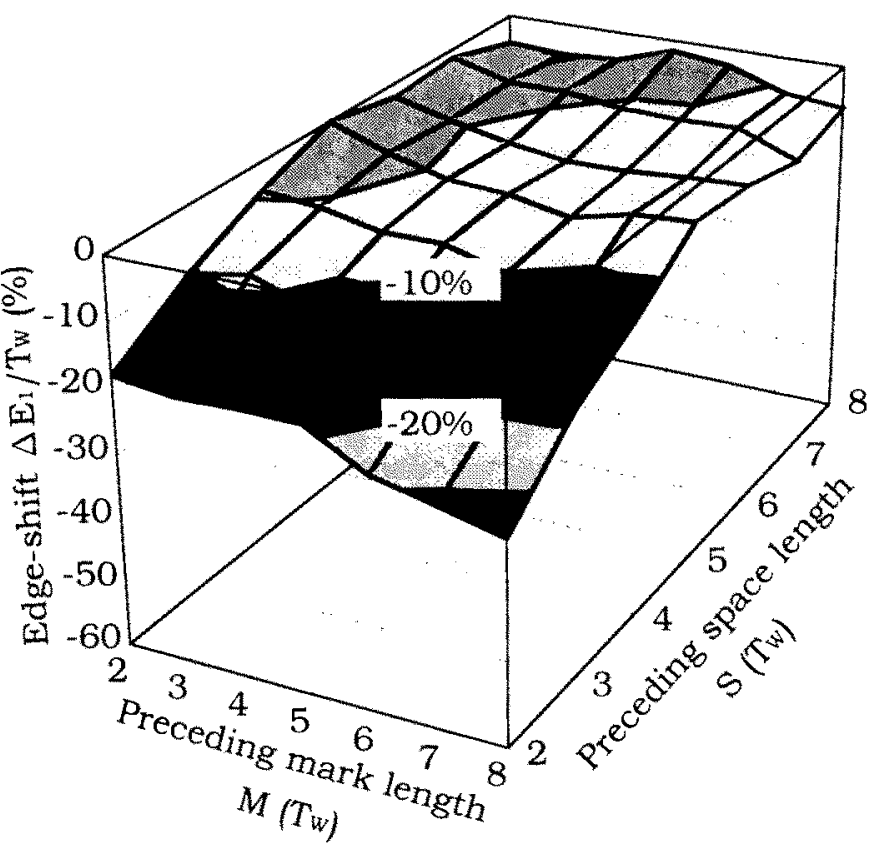

Fig. 7: Leading edge-shift of succeeding mark recorded by ATC waveform. (without equalizer) Edge shifts are generally suppressed. And dependence on preceding mark length is well compensated. 
length) is also evaluated. Results for non-ATC and ATC waveforms are shown in Fig. 6 and Fig. 7, respectively. Edge shifts are generally suppressed and dependence on preceding mark length is well compensated. Figure 4 is the cross section of Fig. 6 and Fig. 7 along the $M$ (preceding mark length) axis at $\mathrm{S}=2$. Other edge-shift behaviors were also measured. Figure 1 shows the dependence on the space length between two marks. And Fig. 1 is the cross section of Fig. 6 and Fig. 7 along $S$ (preceding space length) axis at $\mathrm{M}=2$. Figure 2 shows the dependence of edge-shifts on self-mark length. The edge-shifts without equalization appear almost symmetrical. After optimizing the equalizer, the recording power margin of the ATC waveform is evaluated again. The results are shown in Fig. 8. Here, the recording power margin for the worst pattern is boosted to $25.7 \%$ p-p. The eye-pattern from marks recorded by ATC waveform is shown in Fig. 9. Here, edge-shifts coming from recording process are well canceled. With an optimal equalizer, all edgeshifts are suppressed within $8.4 \% \mathrm{p}$-p of a $0.27-\mu \mathrm{m}$ detection window.

\section{SUMMARY}

Noting the symmetrical edge-shift of ideal marks, we developed a method of optimizing the recording condition and equalizing parameters separately in the system with large ISI. We found out that thermal interference depends not only on the preceding space length but also on the preceding mark length. To suppress this effect, we developed Adaptive Thermo-Compensation, which adaptively compensates for the thermal interference according on the preceding mark length. By ATC, all edge-shifts are suppressed with optimal equalizer within $8.4 \% \mathrm{p}-\mathrm{p}$ of a $0.27-\mu \mathrm{m}$ detection window and, therefore, highly accurate mark-edge controllability is possible.

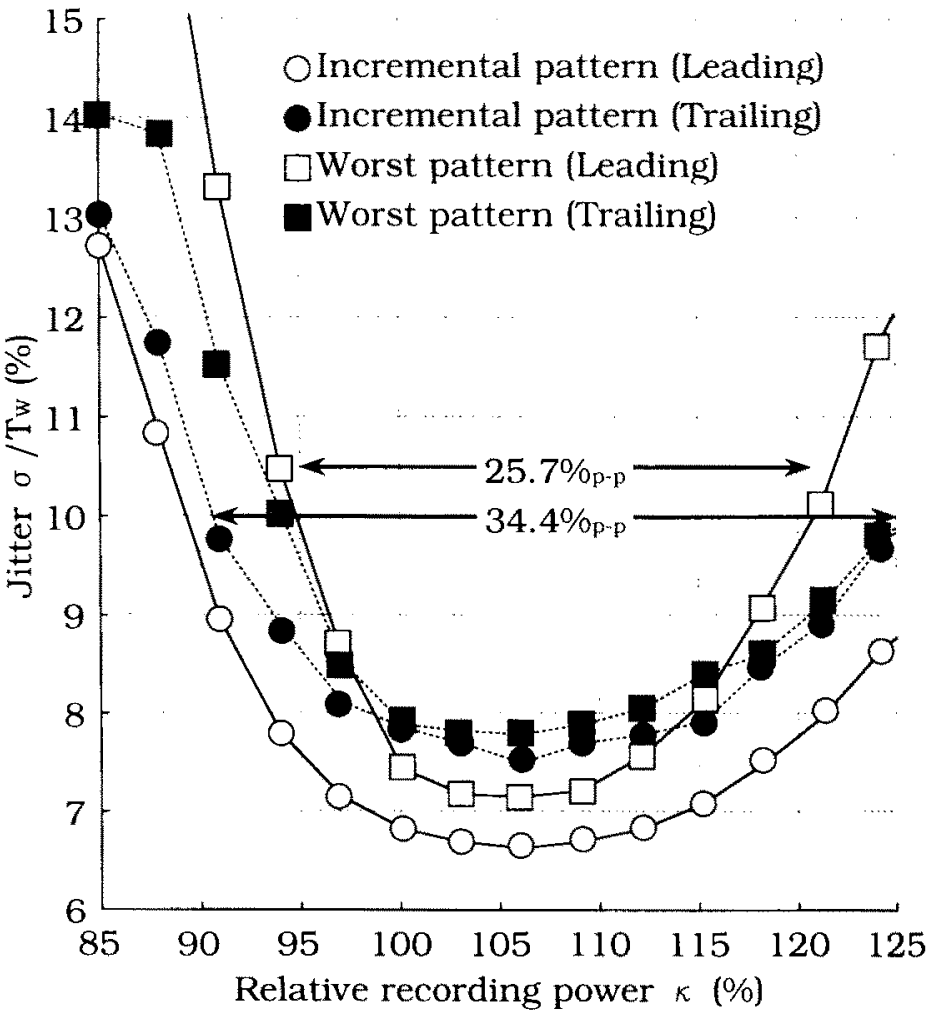

Fig. 8: Recording power margin of ATC waveform. Recording conditions for each edges are matched. Power margin is greatly enhanced.

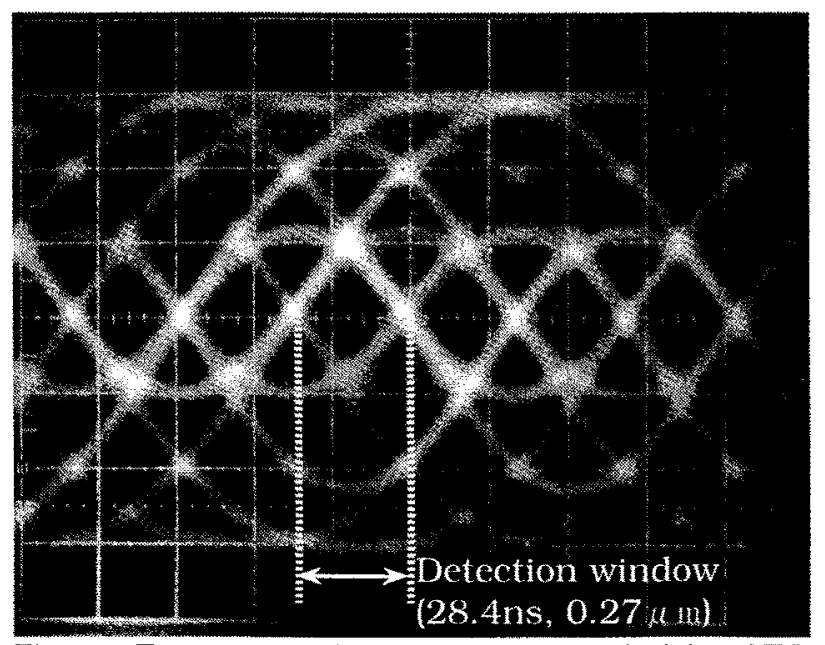

Fig. 9: Eye-pettern from marks recorded by ATC waveform. ( $(v=9.40 \mathrm{~m} / \mathrm{s}$, optimally equalized)

\section{REFERENCES}

[1] A. Saito et al.; Appl. Opt., Vol. 27, No. 20 (1988), p4274

[2] T. Maeda et al.; IEEE Trans. on Mag. Vol. 29. No. 6 (1993), p3787

[3] M. Takahashi et al.; J. Appl. Phys. , Vol. 63, No. 8 (1988), p3838

[4] H. Sukeda et al.; Proc. Int. Symp. on Optical Memory, 1987, Jpn. J. of Appl. Phys., Vol. 26 (1987), Suppl. 26-4, p243 